\title{
A Tangible Platform for Documenting Experiences and Sharing Multimedia Stories
}

\author{
Ali Mazalek and Glorianna Davenport \\ Interactive Cinema Group \\ MIT Media Laboratory \\ 20 Ames Street, E15-368 \\ Cambridge, MA 02139 \\ \{mazalek, gid\}@media.mit.edu
}

\begin{abstract}
Stories are a cultural universal that allow us to reflect on the way we live. Through the act of storytelling, we structure and express our personal experiences and understandings of the world in a form that can be passed on to others - as words, imagery, sounds and gestures. In today's increasingly digital and networked society, we can create media platforms that allow our personal stories to become public and shared. In this paper, we present a tangible platform that has been designed to support the sharing of collaboratively constructed multimedia stories in a social setting. We discuss the way this platform was used in two large-scale personalized storytelling workshops, and examine how it served to engage participants in a self-reflective story revealing process.

\section{Categories and Subject Descriptors}

H.5.2 [Information Interfaces and Presentation]: User Interfaces--input devices and strategies, interaction styles; H.5.1 [Information Interfaces and Presentation]: Multimedia Information Systems-video; H.5.3 [Information Interfaces and Presentation]: Group and Organization Interfaces---collaborative computing; J.5 [Arts and Humanities]; K.3.1 [Computer Uses in Education]: Collaborative Learning
\end{abstract}

\section{General Terms}

Design, human factors

\section{Keywords}

Tangible interface, personal storytelling, co-construction, social interaction, multiple viewpoints, spatial structures

\section{INTRODUCTION}

Everyone is a story maker and a story receiver. In the beginning, most of the stories we make are personal stories. These stories are about our own, everyday life. In making these stories we draw on memory; we reflect, structure and express our stories so that they become public and shared. Occasionally through exceptional

Permission to make digital or hard copies of all or part of this work for personal or classroom use is granted without fee provided that copies are not made or distributed for profit or commercial advantage and that copies bear this notice and the full citation on the first page. To copy otherwise, or republish, to post on servers or to redistribute to lists, requires prior specific permission and/or a fee.

ETP '03, November 7, 2003, Berkeley, California, USA.

Copyright 2003 ACM 1-58113-775-3/03/00011 ..\$5.00.

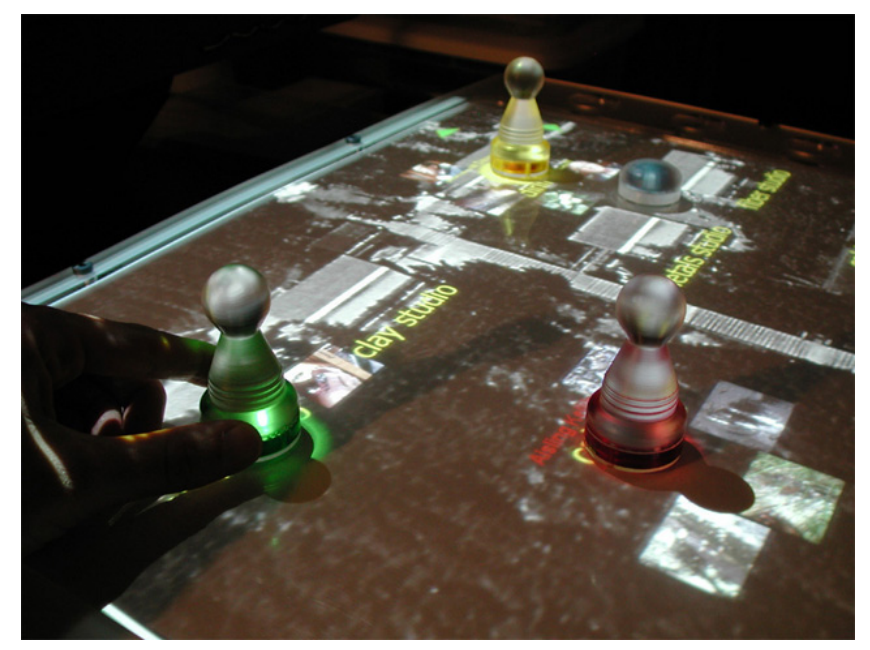

Figure 1. The TViews tangible interaction platform.

effort or recognition of resonance, a personal story becomes reshaped and retold. Ultimately this story may attain a high degree of public exposure.

Personal stories are generally made by someone. Most share a common set of attributes: they operate in one or more locations, they reveal one or more characters, and they incorporate a theme and an objective. In the modern world, electronic media platforms can be designed in ways that allow personal stories to become public and shared for both real and virtual communities.

In particular, opportunities exist to collectively co-construct stories of life experience. In collective storymaking, tangible displays compliment social intention by inviting co-contributors to share in a process of navigating, selecting and experiencing a collection of personal stories. These tangible interface technologies sidestep many of the cumbersome qualities of traditional computer interfaces, allowing story creators to combine rich physical-world interaction spaces with the power and flexibility of multimedia content processing and delivery. As they move out of the computer box and into the physical spaces, surfaces and objects that surround us, digital stories can become an integral part of our everyday lives and face-to-face social interactions.

In this paper we introduce TViews, a tangible platform that supports the sharing of collaboratively constructed multimedia 
stories in a sociable setting. We being with a brief look at some related work, and provide an overview of the system architecture and applications. We then discuss two storytelling workshops that demonstrate how TViews can be used in a real-world setting for the sharing of multiple viewpoint and spatially structured documentary pieces. We conclude with some ideas for how the platform could be used for the development of distributed tangible story spaces.

\section{RELATED WORK}

In 1992, Ed Barrett coined the term sociomedia. In the introduction to his anthology of the same name, Barrett writes: "Sociomedia suggests that computer media exist for 'social' purposes: as a means to objectify, exchange and collaborate, invoke, comment upon, modify, and remember thoughts and ideas." ([1], p.1) He goes on to explain that in designing computer media we are essentially creating a mechanism for the social construction of knowledge.

In bringing storymaking together with real social interaction around the TViews platform, our research on personal narrative augments the discourse in social computing and group reflection, as well as in navigable story systems. This section takes a brief look at some of the related work in these areas.

The original Domesday Book was commissioned by William the Conqueror, who invaded England in 1086. Twenty years later the first draft of the book was complete, and contained records for some 13,418 settlements in the English counties south of the Scottish border. This original census-like survey served as inspiration for an electronic project done in the mid 1980s by BBC Interactive that focused on community memory [4]. In two special issues of Interacting with Computers, Stathis and Purcell identify a number of projects that were designed to link real communities through storytelling, memory and a range of specially designed applications that promoted social activity $[8$, 9].

Online weblogs permit a diary approach to making and reflecting on stories about almost anything. In 2003, this early blog culture is again transforming itself to support multiple authors in the creation of semi-coherent stories around topics of particular interest. The Digital Dialogues weblog, built and maintained by Aisling Kelliher, is a co-constructed diary of symposium events that was designed to complement the spatial/tangible display discussed in this paper $[3,5]$.

Many approaches to storytelling engines have engaged audiences in personalized story experiences. The Contour and Dexter systems use a spreading activation network model to present meaningful and continuous story sequences based on system knowledge of content and user interaction [7]. Agent Stories uses software agents with specific behavioral traits to construct different variations of a tale from a pre-constructed web of story material [2].

\section{THE TVIEWS PLATFORM}

TViews is a tangible display platform that supports interactive story navigation through the manipulation of graspable pawns and other tangible tools on a sensing surface. This section gives an overview of the interface architecture, and describes two

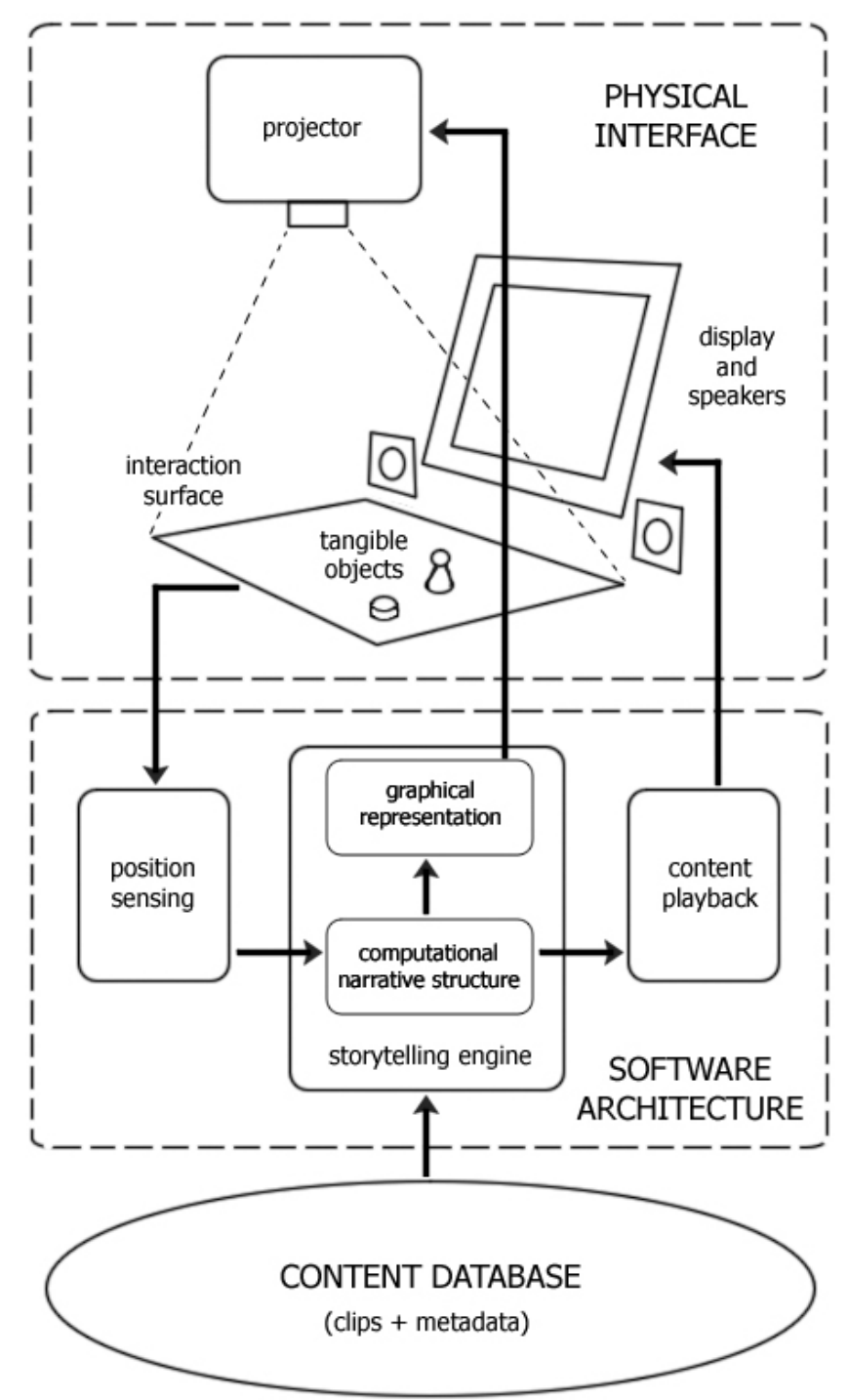

Figure 2. TViews system diagram.

storytelling applications that have been written for the interface: a narrative engine for multi-viewpoint character-driven stories, and a navigation system for spatially distributed collective stories.

\subsection{Interface Architecture}

TViews couples a sensing platform and graphical projection with a standard on-screen display and speaker setup. The interaction surface uses electromagnetic field sensing technology to enable the tracking of tagged physical objects as they are moved around the two-dimensional horizontal plane. Synchronized graphics are projected onto the objects to provide a visual representation of the current state of the story. As such, the tangible objects act as both input and output for the storytelling system. The on-screen display and speakers can be used to playback story content in audio-visual form or to give additional story information, such titles or textual descriptions. The top portion of figure 2 illustrates the physical setup of the TViews platform. 


\subsection{Application Overview}

The TViews software architecture consists of three independent modules, illustrated in the middle section of figure 2 . The modules are implemented in Java.

The position sensing module uses a polling mechanism to gather resonant frequency information about each tag. This data is transmitted to the computer at 57,6 kbaud via the RS-232 protocol, where it is decoded into $(\mathrm{x}, \mathrm{y})$ positions for each tangible object.

The playback module is responsible for playing content clips in the form of video, audio, images or text to the on-screen display and speakers. The implementation makes use of Apple's QuickTime for Java API.

The storytelling engine module forms the core of the system's interactive functionality and determines how the audience will perceive and manipulate story content on the TViews interaction surface. It consists of a computational narrative structure, which provides a means of organizing the story information from the content database, and a graphical engine, which determines how the story content will be visually represented through graphical projection. Changes in the story space based on user interactions are reflected by dynamic changes in the projected graphics. By separating the storytelling engine from the position sensing and content playback modules, TViews becomes a flexible story platform that can be used to drive a variety of different applications. So far we have explored multi-viewpoint and spatial approaches to story revealing.

\subsubsection{Tangible Viewpoints}

Tangible Viewpoints allows the audience to experience characterdriven narratives. The content clips of a multi-viewpoint story are organized according to the character viewpoint they represent, and their place in the overall flow of the narrative. When a pawn is placed on the interaction surface, the story clips associated with its character viewpoint are projected around it in the form of small thumbnails. These can be activated using a small lens-shaped selection tool, causing the narrative to advance and new clips to become available. Activated clips are also sent to the playback module, and in turn to the on-screen display.

The computational story engine moves the story forward as the audience interacts. It makes use of a rule-based structure for controlling narrative progression, and a spreading activation network to ensure continuity and relevance as new content clips are revealed to the audience. The details of the implementation are described in [6].

\subsubsection{Tangible Spatial Narratives}

Through human actions, conversations, and the creation of memories, physical spaces are transformed into social constructs and can become the arena for story construction and narrative development. In this application, we explore how such spaces can be created in a tangible form by allowing audience members to navigate through complex spatially structured and co-constructed documentary works.

Content clips are organized according to location, character and time, and a visual landscape is projected onto the interaction surface to provide a spatial framework for the overall story and the many perspectives and narrative threads that emerge as it unfolds. Pawns can be associated to different characters in the

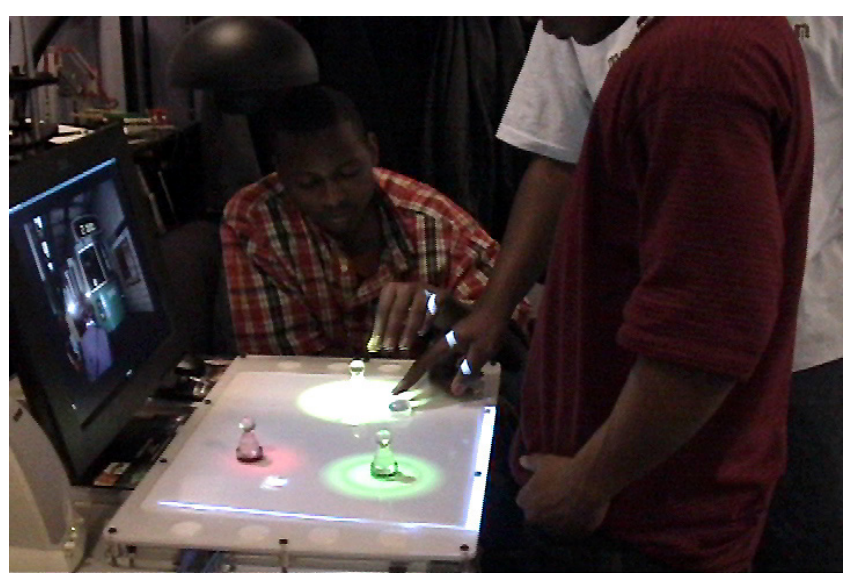

Figure 3. Clubhouse members engaging with the Tangible Viewpoints storytelling application.

story, and moved around the map in order to reveal content clips in the form of small thumbnails. These content clips are dynamically retrieved from the story database through character and location-based queries that are determined by the placement of pawns on the interaction surface. A clock tool can be used to retrieve clips using the additional parameter of time. Content clips can be played on the display screen by gliding the lens-shaped selection tool over top of the thumbnails on the interaction surface.

\section{STORYTELLING EXPERIENCES}

The TViews platform can be used to organize and convey the stories we create about our personal experiences and perspectives on life to small groups of people in an informal and social setting. As such, the tool serves as a space for story and knowledge exploration. By sharing their stories in a multimedia form, authors and audiences can engage in a reflective learning process, reexamining their own experiences and relating them to the perspectives and experiences of others.

To date, the TViews multi-viewpoint and spatial applications have been used to support two different large-scale personalized storytelling experiences, in which the community of story authors were also invited to become the audience members of their own stories. Through iteration between story creation and story engagement, participants were able to watch their own story grow and evolve, and as a result became engaged in an active process of story revealing and personal reflection. The following sections provide an overview of these two storytelling workshops.

\subsection{Computer Clubhouse Workshop}

In November 2001 we conducted a 10-day storytelling workshop at the Boston Museum of Science Computer Clubhouse. This after school learning environment provides a safe space for young people from underserved communities to engage in creative activities and skill building through the use of technology. Three teenage participants were selected to create personal stories about their lives in Boston that would be played back using the Tangible Viewpoints system.

During initial brainstorming sessions, the participants worked together to develop a structure for their story. Their goal was to create a documentary piece that would chronicle one day in each 
of their lives in their East Boston community. Participants used digital still cameras to record images for their stories. They also provided voice-overs and metadata information before adding their clips to the content database.

The TViews platform was set up in a central area of the Clubhouse. Workshop participants and other members from the community were free to interact with the story as it evolved over the course of the 10 days. Through social interaction and discussion around the tangible story platform, the storytelling process became a collaborative and iterative activity. Workshop participants drew on the skills and ideas of their fellow Clubhouse members, and incorporated them into the creation of their own personal stories.

While the creation of individual story threads allowed them to share personal perspectives on life in their East Boston community, participants found that their stories were not entirely separate. Their stories intersected at certain points, for instance when their activities coincided at school or in the clubhouse. These moments revealed the way in which their interpretations of events often differed from the interpretations of those around them. By providing a shared framework for the telling of personal stories, participants were able step back and examine their own lives and experiences from a broader perspective. In this way, they could begin to understand how their own stories formed a part of the larger collective story of their community.

\subsection{Digital Dialogues Symposium}

In September 2002 we organized a storytelling workshop for the Digital Dialogues: Technology and the Hand symposium held at the Haystack School of Mountain Crafts in Maine in collaboration with the MIT Media Laboratory. The symposium focused on the creation of artistic pieces in a studio-based environment, and the 65 participants were invited to use their hands in collaboratively forming materials, media, and ideas. The goal was to explore how technology and handcraft can be brought together, and to initiate dialogues and an exchange of expertise between these two traditionally separate communities. The storytelling workshop enabled participants to document the events taking place across the different craft studios over the course of the symposium. The documentary took the form of a living record - a piece that grew as the symposium progressed, and incorporated the different perspectives of the large number of people coming from a wide variety of backgrounds.

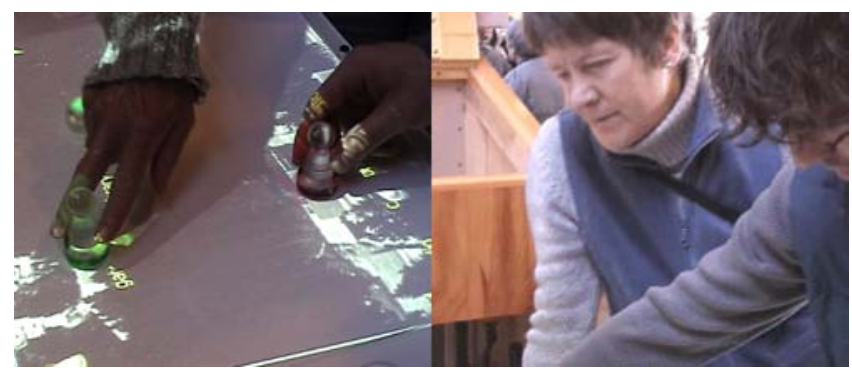

Figure 4. Symposium participants engaging with their stories using the TViews platform.

The story of the symposium progressed along three main axes: space (the symposium was spatially distributed across the Haystack campus and craft studios), time (the event lasted 3-4 days), and character viewpoint (the many symposium participants provided widely differing personal perspectives on the unfolding events). Based on these parameters, we provided a core narrative framework for the piece. Participants used digital video and still cameras to record their activities and experiences in the studio spaces. These clips were then annotated with the appropriate metadata and loaded into a database that could be queried according to different keywords or combinations of keywords.

Over the course of the symposium, participants used the TViews platform to collaboratively engage with their story as it was still being created. The map of the Haystack campus was projected onto the sensing surface and provided a spatial framework for viewer interactions. This spatial layout helped ground the digital story clips in relation to the actual events taking place in the real world. Viewers could associate the graspable pawns to different symposium participants, moving them around the studio spaces depicted on the map. They could also use a clock tool to navigate back and forth through time. In this way, they could follow and compare the activities of different participants across both space and time.

As people experimented with the tangible interface, their engagement with the video clips was enhanced by the fact that they were familiar with the people and events portrayed. The video clips presented an opportunity for many of the craftspeople to watch themselves actively engaged in their craft for the first time, and the social nature of the tangible platform allowed them to share and reflect on this experience with others. The collaborative exploration of their own evolving story fostered an exchange of ideas that participants could immediately bring back into the work environment and incorporate into the next iteration of story creation. As such, the movement between story creation and engagement became a cyclical process, with each part informing the evolution and meaning of the next.

\section{DISTRIBUTED STORY SPACES}

At its current scale, the TViews platform works best for small groups of audience members interacting at a time. Moreover, given that only a single instance of the platform exists, the stories created for it can only ever attain a limited level of public exposure.

We have devised various ways to circumvent this limitation. In the Tangible Viewpoints application, viewers can save linear versions of their paths through the story space as QuickTime movies. While these short segments can give viewers something to take away with them, it also removes the movies from their often still-evolving larger context. In the Digital Dialogues piece, an online weblog [3] was created to complement the tangible display, and supports continuing online discussions around the story material.

For the future, we envision connecting multiple distributed tangible displays in order to support a larger community of audience. In this case, the story database would reside on a central server and each instance of the TViews platform would act as a kind of window onto the story world. This concept of distributed tangible story spaces opens up a variety of new possibilities for remote collaborative storytelling. Audience members interacting in one location might save their particular view on the story world and send it to other platforms across the network. Given the flexibility of the computational story structure, new content clips 
could be uploaded to the central server from any location and at any time. These changes in the content database could be broadcast throughout the network of display platforms, which would guide audience members to explore the new story threads in the system. The network-based system might also allow several displays to synchronize their states, enabling remote groups of audience members to share their experiences in engaging with the story in real-time.

\section{CONCLUSION}

In this paper we present a tangible platform for the sharing of documentary stories, and discuss its use in two large-scale collaborative storytelling events based on multi-viewpoint and spatial approaches to narrative interaction. These workshops demonstrate how personalized storytelling experiences can engage audiences in a self-reflective story understanding process. Through iterative cycles of story creation and collaborative story revealing, participants were able to examine their own evolving story threads in relation to the larger story of their community. The lessons learned in this process and through their conversations around the tangible platform could be immediately incorporated into their lives and into the telling of their future stories.

While tangible interfaces can help engage audiences in a collaborative story revealing activity, they can also limit the level of public exposure of a particular community's stories. In looking forward, we can try to think of ways of making these displays available to a wider group of distributed audience members who might be able to share their stories remotely.

\section{ACKNOWLEDGMENTS}

Many people have contributed to this work. We would like to thank the members of the Interactive Cinema group, as well as the MIT Media Laboratory and its sponsors. We would also like to thank all those who participated in and helped support the storytelling workshops at the Computer Clubhouse and at the Digital Dialogues symposium.

\section{REFERENCES}

[1] Barrett, Edward (Ed.). Sociomedia: Multimedia, Hypermedia, and the Social Construction of Knowledge. MIT Press, Cambridge, MA, November 1992.

[2] Brooks, Kevin. Metalinear Cinematic Narrative: Theory, Process, and Tool. Ph.D. Thesis, MIT Media Laboratory, 1999.

[3] Digital Dialogues Video Weblog (Accessed September 2003). http://weblogs.media.mit.edu/digitaldialogues/

[4] Finney, Andy. The Domesday Project - November 1986. Online artile at http://www.atsf.co.uk/dottext/domesday.html (Accessed October 2003).

[5] Kelliher, A., Mazalek, A., Davenport, G. "Documenting Digital Dialogues: Engaging Audience in the Construction of a Collective Documentary Across Space and Time" in Proceedings of TIDSE '03, Springer-Verlag, pp.248-259.

[6] Mazalek, A., Davenport, G., Ishii, H. "Tangible Viewpoints: A Physical Approach to Multimedia Stories " in Proceedings of ACM Multimedia '02, ACM Press, 2003, pp.153-160.

[7] Murtaugh, Michael. The Automatist Storytelling System. MS Thesis, MIT Media Laboratory, 1996.

[8] Stathis, K., Purcell, P. "Intelligence and Interaction in Community-Based Systems" in Interacting with Computers. Vol. 14, No. 6, December 2002, pp.639-642.

[9] Stathis, K., Purcell, P. "Intelligence and Interaction in Community-Based Systems (Part 2)" in Interacting with Computers. Vol. 15, No. 1, January 2003, pp.103. 